\title{
Social group behaviour of triads. Dependence on purpose and gender
}

\author{
Francesco Zanlungo ${ }^{1}$, Zeynep Yücel $^{2}$, Takayuki Kanda ${ }^{1,3}$ \\ ${ }^{1}$ ATR IRC \\ Kyoto, Japan \\ zanlungo@atr.jp,kanda@atr.jp, \\ ${ }^{2}$ Okayama University \\ Okayama, Japan \\ zeynep@okayama-u.ac.jp \\ ${ }^{3}$ Kyoto University \\ Kyoto, Japan
}

\begin{abstract}
We analysed a set of uninstructed pedestrian trajectories automatically tracked in a public area, and we asked a human coder to assess their group relationships. For those pedestrians who belong to the groups, we asked the coder to identify their apparent purpose of visit to the tracking area and apparent gender. We studied the quantitative dependence of the group dynamics on such properties in the case of triads (three people groups) and compared them to the two pedestrian group case (dyads), studied in a previous work. We found that the group velocity strongly depends on relation and gender for both triads and dyads, while the influence of these properties on spatial structure of groups is less clear in the triadic case. We discussed the relevance of these results to the modelling of pedestrian and crowd dynamics, and examined the possibility of the future works on this subject.
\end{abstract}

Keywords: group behaviour, social relations, dyads, triads

\section{Introduction}

Researchers in the fields of pedestrian behaviour and crowd modelling have become recently more and more aware of the necessity of studying and modelling the behaviour of social groups. Groups represent an important, if not predominant, portion of urban pedestrian crowds [1,2], and they exhibit groupspecific behaviour $[3,4,5,6]$ and thus understanding and modelling them is necessary to reproduce crowd behaviour in normal and emergency conditions [7]. As a result, a few statistical studies, mathematical and numerical models of group behaviours have been developed [3,8,9,10,11,12,13,14]. It is nevertheless clear that, being related to human social behaviour, group dynamics is probably influenced by the composition of the group and by the relation between the members $[6,15,16]$.

In [17], we studied the dependence of dyad group dynamics on purpose, relation, gender, age and height, along with the interplay of these factors. Our analysis was able to quantitatively confirm some intuitive properties, such as a larger interpersonal distance and walking velocity in workers compared to leisure oriented pedestrians, in colleagues compared to friends and couples, and in males compared to females. Groups including tall pedestrians were also observed to walk faster, as did groups composed of active age adults, when compared to groups with children or elderly people. More surprising results were related to the behaviour of families, which appeared to walk in a less organised (non-abreast) way than other groups, and exhibit counter-intuitive features such as walking faster in high density settings. This behaviour was found to be due to the irregular motion of children.

In this work we extended the statistical analysis performed in [17] on dyads to groups formed by three people. 


\section{Data set and definitions}

Our analysis is based on a data set of trajectories of unconstrained and uninstructed groups, moving in an "ecological" environment (a building in Osaka, Japan, where shopping areas, offices, a monorail station and a ferry terminal are present). The pedestrian trajectories (including pedestrian height) have been automatically tracked using 3D range sensors, while group relation and group composition have been manually determined by human coders through video analysis (see $[4,17]$ for the details of the tracking system and the reliability of human coding, i.e. agreement between coders).

In the current work, we focused on the behaviour of triads. Following [17,18], we defined and analysed their intrinsic properties as

- purpose of movement

- relation between the members

- $\quad$ gender of the members

- age of the members

- height of the members

Regarding the purpose, we divided the groups in the work oriented and leisure oriented classes; regarding the relation in colleagues, families and friends (couples, analysed in [17], are absent in triads); regarding the gender we considered the number of females (from 0 to 3); while regarding the age and the height we analysed the average, minimum and maximum value assumed by these properties in each group. Nevertheless, due to the limited space, in this work we will show only the results concerning the purpose and gender, which are of relatively easy interpretation and comparison to the dyadic case.

In order to analyse in a quantitative way the triad dynamics, we first, following [17], defined the group velocity as

$$
\mathbf{V}^{g 3}=\sum_{i} \mathbf{v}_{i} / 3
$$

and used a reference frame whose $y$ axis is aligned with $\mathbf{V}^{g 3}$ to measure the components $\left(x_{i}, y_{i}\right)$ of all pedestrians (Figure 1 left). The labels are chosen in such a way that $x_{1} \leq x_{2} \leq x_{3}$.
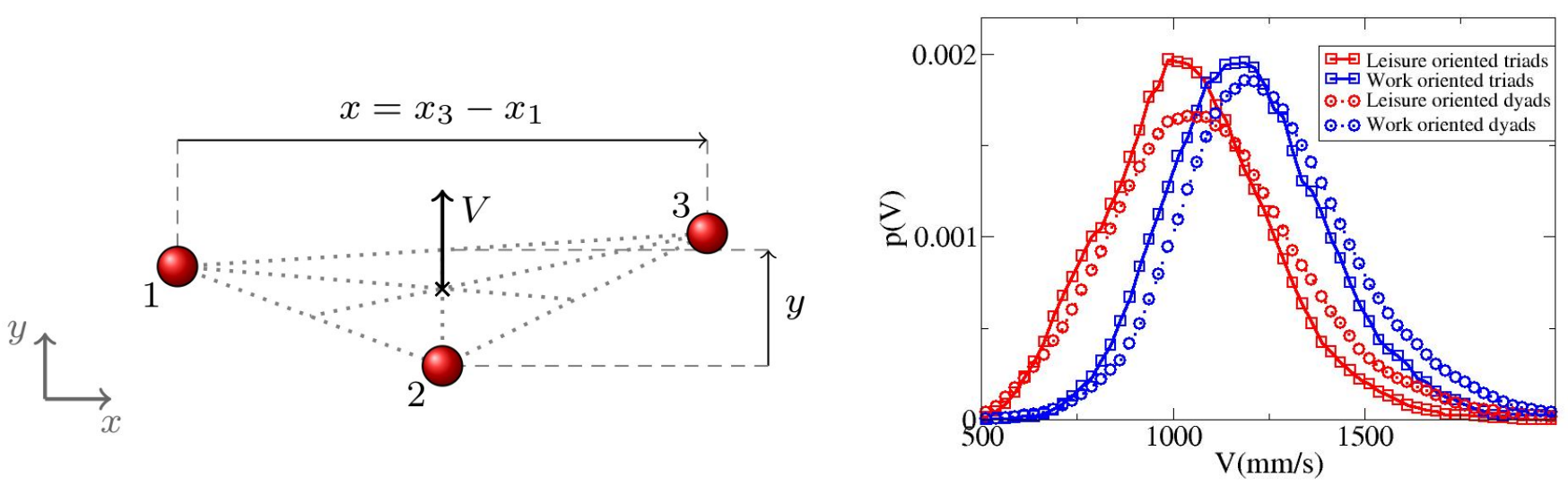

Figure 1: Left: observable definition. Right: comparison between triadic and dyadic $V$ probability distribution function dependence on purpose. 
Our quantitative analysis will be based on the following observables:

1. group velocity, defined as $V^{g^{3}}=|\mathbf{V}|$,

2. group width, defined as $x^{g 3}=x_{3}-x_{1}$,

3. group depth, defined as $y^{g 3}=\left|y_{3}+y_{1}-2 y_{2}\right| / 2$.

In the previous equations, the superscript $g 3$ is used to differentiate these observables from their two people equivalent, defined and analyzed in [17] as

1. group velocity, defined as $V^{g 2}=\left|\sum_{i} \mathbf{v}_{i} / 2\right|$,

2. group width, defined as $x^{g 2}=x_{2}-x_{1}$,

3. group depth, defined as $y^{g 2}=\left|y_{2}-y_{1}\right|$.

In [17], we also analysed the distance between pedestrians $r^{g 2}$, but such a quantity has not a straightforward triadic generalisation and will not be analysed in this work.

For each observable, we provided the number of groups analysed $N$, its average value, standard deviation and standard error. We compared the values assumed between different classes giving an ANOVA based "statistical significance" $p$ value (strongly dependent on the number of groups observed) along with an effect size [17] (comparing average value difference to standard deviations, not strongly influenced by the number of observations). We also showed for each observable and class the overall empirical probabilistic distribution functions (pdf), to compare actual variable distribution beyond their average values.

\section{Results concerning purpose}

Table I shows the observable dependence on the purpose classes in triads, while the results for dyads are copied from [17] and reported in Table II. A standard ANOVA shows that the influence of purpose on velocity is extremely significant, the one on $x$ is still significant but reduced with respect to $V$, while no significant effect is present for $y$. In dyads, the purpose had a significant effect on all variables (particularly strong on $V$ and $x$ ), although the analysis was based on a larger data set. To correct for the difference in sample size, we may observe that the effect size analysis shows again a large effect on velocity

TABLE II

OBSERVABLE DEPENDENCE ON PURPOSE FOR DYADS. LENGTHS IN MILLIMETRES, TIMES IN SECONDS.

\begin{tabular}{|c|c|c|c|c|}
\hline Purpose & $N$ & $V^{g 2}$ & $x^{g 2}$ & $y^{g 2}$ \\
\hline Leisure & 716 & $1118 \pm 7.3(\sigma=195)$ & $628 \pm 6.1(\sigma=162)$ & $383 \pm 12(\sigma=334)$ \\
\hline Work & 372 & $1271 \pm 8.2(\sigma=158)$ & $713 \pm 8(\sigma=154)$ & $332 \pm 15(\sigma=289)$ \\
\hline$p$ & & $<10^{-8}$ & $<10^{-8}$ & 0.0126 \\
\hline effect size & & 0.832 & 0.533 & 0.16 \\
\hline \multicolumn{5}{|l|}{} \\
\hline$p$ & & $<10^{-8}$ & 0.0248 & 0.818 \\
\hline effect size & 1.01 & 0.363 & 0.037 \\
\hline
\end{tabular}

(even stronger than the one on dyads), a reduced one on $x$ and a negligible one on $y$. 
A direct comparison of the triad and dyad probability distribution functions for the $V$ observable is shown in Figure 1 (right), while the corresponding distributions for $x$ and $y$ are shown in Figure 2. The effect of the purpose on velocity appears indeed to be very similar in triads and dyads, with a shift between the leisure and work distribution of roughly $150 \mathrm{~mm} / \mathrm{s}$ regardless the number of the pedestrians in the group, as can be verified from the average values in Tables I and II (for an explanation of the difference in velocity between dyads and triads, refer to [3]). The comparison of $x$ and $y$ pdfs between triads and dyads is obviously less straightforward, since these quantities roughly scale with the group size. Nevertheless, we may see that the tendency of workers to assume a larger abreast distance, that was observed in dyads (roughly $80 \mathrm{~mm}$ more, according to table 2), is confirmed also in triads (roughly $100 \mathrm{~mm}$ more according to table 1). The $y$ distribution for dyads suggested that workers have a more regular abreast configuration than leisure oriented pedestrians (higher peak), while in the triadic case we were not able to observe any clearly different pattern.
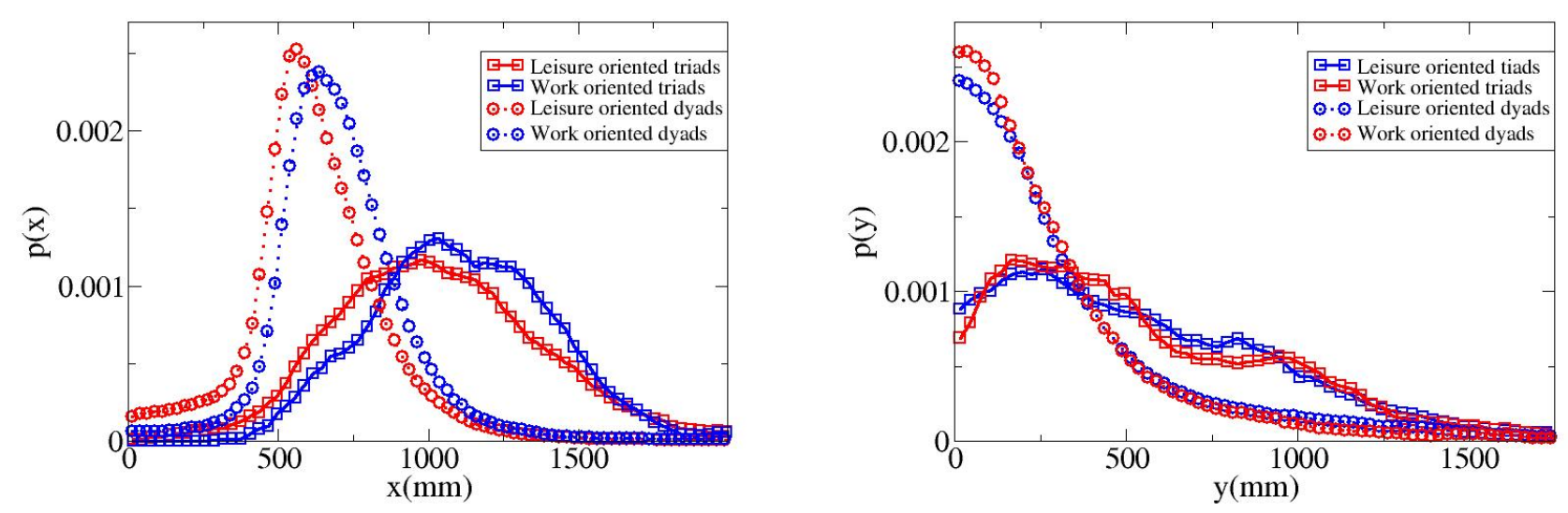

Figure 2: Left: comparison between triadic and dyadic $x$ probability distribution function dependence on purpose. Right: equivalent comparison for the $y$ pdf.

\section{Results concerning gender}

Table III shows the observable dependence on the gender, while the results for dyads are copied from [17] and reported in Table IV. The ANOVA shows again a very strong statistical significance for the effect of gender on velocity, while no statistical significance is observed for the spatial observables, in contrast to the dyadic case, in which all observables presented statistically significant differences due to the gender of the groups. As previously discussed, these results may be strongly influenced by the smaller statistical sample used for triads. For this reason, we analysed also the effect size, which shows again that the effect on velocity is much stronger than that on spatial observables (and, again, even stronger than in the dyadic case). Furthermore, the effect size on $x$ is strongly reduced in triads (while that on $y$ is similar on triads and dyads). 
TABLE III

OBSERVABLE DEPENDENCE ON GENDER FOR TRIADS. LENGTHS IN MILLIMETRES, TIMES IN SECONDS.

\begin{tabular}{|c|c|c|c|c|}
\hline Gender & $N$ & $V^{g 3}$ & $x^{g 3}$ & $y^{g 3}$ \\
\hline Three females & 44 & $1060 \pm 21(\sigma=140)$ & $1030 \pm 39(\sigma=256)$ & $565 \pm 51(\sigma=338)$ \\
\hline Two females & 26 & $1008 \pm 28(\sigma=142)$ & $1087 \pm 57(\sigma=289)$ & $524 \pm 76(\sigma=386)$ \\
\hline Two males & 27 & $1053 \pm 29(\sigma=152)$ & $1028 \pm 45(\sigma=236)$ & $621 \pm 47(\sigma=245)$ \\
\hline Three males & 72 & $1210 \pm 19(\sigma=165)$ & $1093 \pm 34(\sigma=285)$ & $602 \pm 41(\sigma=352)$ \\
\hline$p$ & & $<10^{-8}$ & 0.545 & 0.696 \\
\hline effect size & & 1.26 & 0.237 & 0.303 \\
\hline
\end{tabular}

TABLE IV

OBSERVABLE DEPENDENCE ON GENDER FOR DYADS. LENGTHS IN MILLIMETRES, TIMES IN SECONDS.

\begin{tabular}{|c|c|c|c|c|}
\hline Gender & $N$ & $r^{g^{2}}$ & $x^{g^{2}}$ & $y^{g^{2}}$ \\
\hline Two females & 252 & $1102 \pm 12(\sigma=193)$ & $647 \pm 7.8(\sigma=123)$ & $321 \pm 20(\sigma=311)$ \\
\hline Mixed & 371 & $1111 \pm 9.5(\sigma=183)$ & $613 \pm 9(\sigma=174)$ & $416 \pm 18(\sigma=350)$ \\
\hline Two males & 466 & $1254 \pm 8.3(\sigma=178)$ & $699 \pm 7.7(\sigma=166)$ & $349 \pm 14(\sigma=293)$ \\
\hline$p$ & & $<10^{-8}$ & $<10^{-8}$ & 0.000484 \\
\hline$\delta$ & & 0.825 & 0.51 & 0.282 \\
\hline
\end{tabular}
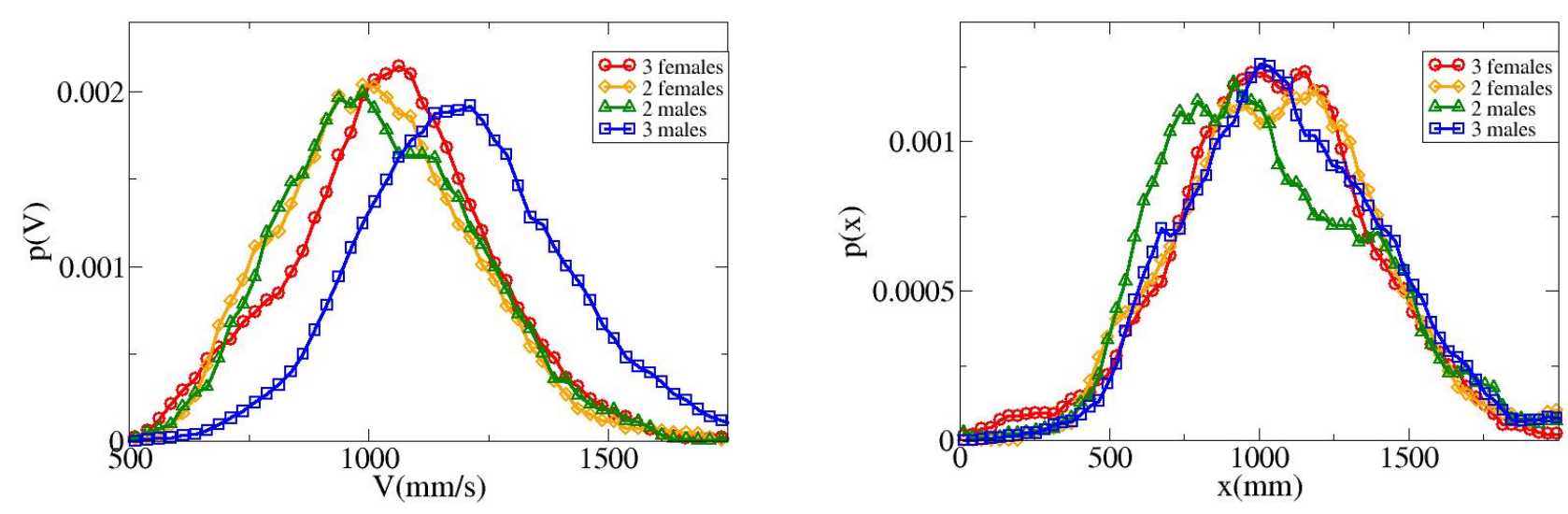

Figure 3: Left: dependence of $V^{g 3}$ (triads) pdf on gender. Right: dependence of $x^{g 3}$ (triads) pdf on gender.

The dependence of triadic probability distribution functions on gender is shown in Figures 3-left, 3-right and 4-left for, respectively, the $V, x$ and $y$ observables. The comparison to the dyadic case is limited, for simplicity's sake, to same gender groups (i.e. 2 females or 2 males for dyads, 3 females or 3 males for triads) and shown in Figures 4-right, 5-left and 5-right for, respectively, the $V, x$ and $y$ observables. 

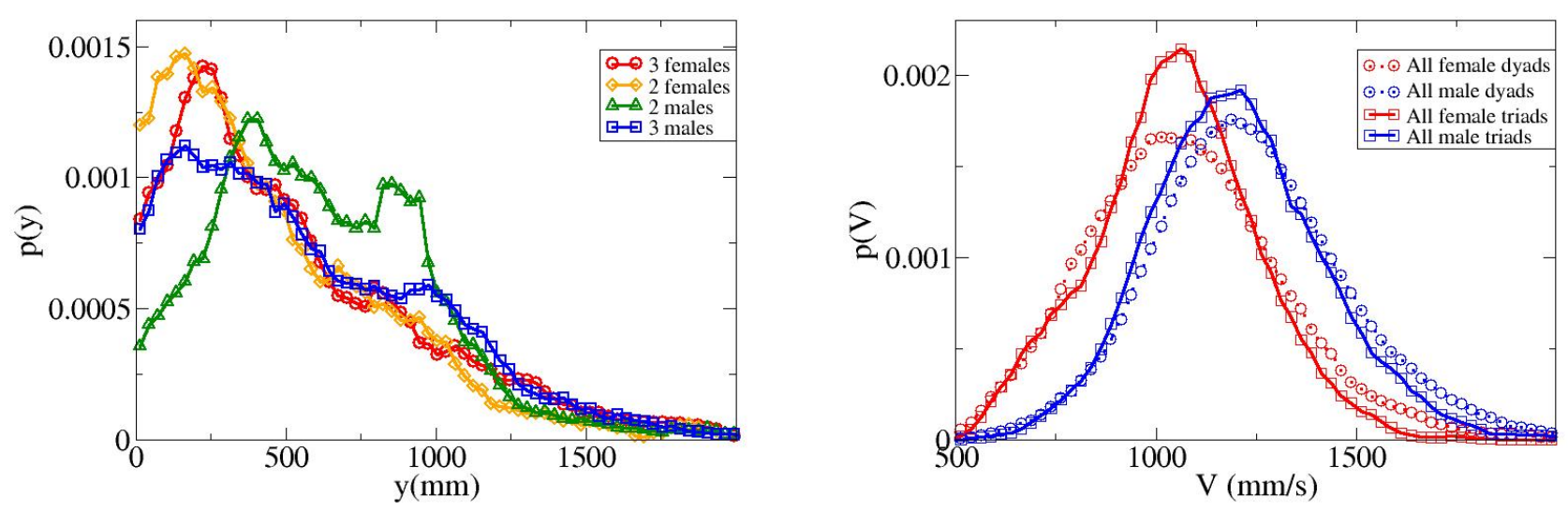

Figure 4: Left: dependence of $y^{g 3}$ (triads) pdf on gender. Right: Comparison between triadic and dyadic $V$ pdf dependence on gender.
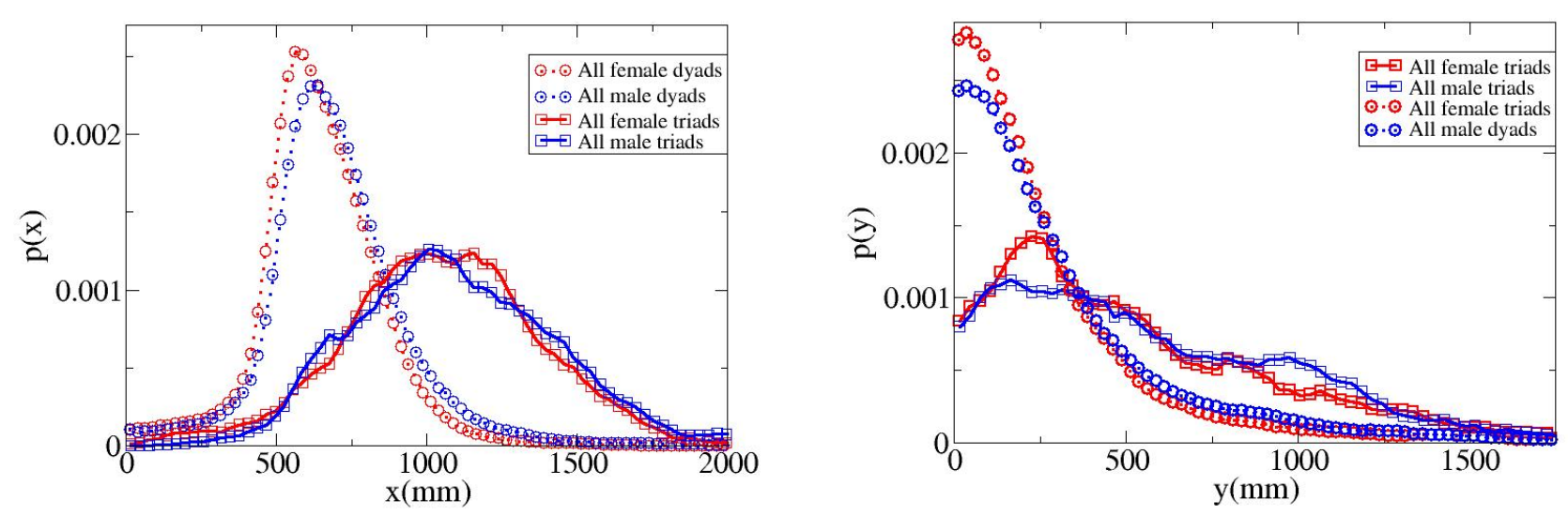

Figure 5: Left: Comparison between triadic and dyadic $x$ pdf dependence on gender. Right: Comparison between triadic and dyadic $y$ pdf dependence on gender.

Both from Figure 4-right and Tables III and IV, we may see that males walk roughly $150 \mathrm{~mm} / \mathrm{s}$ faster than females regardless of group size (dyads being again faster than triads of $40 \mathrm{~mm} / \mathrm{s}$ ). On the other hand, the tendency of females to walk closer in abreast distance (Figure 5-left), and males in more ordered way (Figure 5-right) is observed in dyads but not in triads. 
Concerning the behaviour of mixed gender triads, we may, from the qualitative analysis of the distribution functions, see that they appear to walk slightly slower than female groups (Figure 3-left), and that the spatial distribution of groups with two males appears to have features clearly different from the others. As discussed in the conclusions, without expanding the sample and studying in more detail the relation and age properties of these groups, it is impossible to understand the meaning and significance of these observations.

\section{Conclusions}

We have observed for triads the same velocity patterns (larger in workers and males) as the patterns observed for dyads, and with similar effect size and statistical significance. While not explicitly reported in this work, we also observed that the effect of relation, age and height on velocity is very similar between two people and three people groups. For dyads we observed also clear patterns in spatial observables, namely males walked at a larger abreast distance and in a more ordered abreast configuration (narrower $y$ distribution). While the effect of purpose on $x$ was observed also for triads, evidence confirming the other effects has not been found. These observations seem to suggest that the geometrical shape of three people groups (V formation, [3]) has some specific features that are more resilient to differences in intrinsic group properties than the simple abreast walking of dyads. An alternative explanation may be that dyadic interactions are of stronger nature than triadic ones, and thus affect more the geometry of groups. Furthermore, triads, by being spatially larger, may suffer more the influence of the surrounding environment (other pedestrians and architectural structures), and as a result the effect of intrinsic features may be reduced.

In [17] we provided a cross-analysis of the effect of different intrinsic features on group behaviour. For example, we observed that although, in our observed sample, work oriented dyads are mainly composed of two males, the effect of gender and purpose were still present when isolated (e.g. comparing two female worker groups to two male ones). Furthermore, we verified that some specific features of mixed gender groups were better explained when couples were isolated from parent-child dyads. A similar analysis will be extremely useful also in the triadic case, but in order to provide it, we will need to expand our statistical sample.

The findings of this work, in particular if confirmed and better elucidated by the analysis of larger set of triads, may help in the development of better group models and, as a consequence, crowd simulators.

\section{Acknowledgments}

This work is based on results obtained from a project commissioned by the New Energy and Industrial Technology Development Organization (NEDO).

\section{References}

1. M. Schultz, L. Rößger, F. Hartmut and B. Schlag, Group dynamic behavior and psychometric profiles as substantial driver for pedestrian dynamics, in Pedestrian and Evacuation Dynamics 2012, U. Weidmann, U. Kirsh and M. Schreckenberg, Vol II, pp. 1097-1111 (2014) .

2. M. Moussaïd M, N. Perozo, S. Garnier, D. Helbing, and G. Theraulaz, The walking behaviour of pedestrian social groups and its impact on crowd dynamics, PLoS One, 2010, 5, 4, e10047.

3. F. Zanlungo, T. Ikeda, and T. Kanda, Potential for the dynamics of pedestrians in a socially interacting group, Physical Review E, 2014, 89, 1, 021811.

4. F. Zanlungo, D. Brščić and T. Kanda, Spatial-size scaling of pedestrian groups under growing density conditions, Physical Review E 91 (6), 062810 (2015)

5. F. Zanlungo and T. Kanda, A mesoscopic model for the effect of density on pedestrian group dynamics Europhysics Letters, 111, 38007 (2015). 
6. L. He, J. Pan, W. Wang and D. Manocha, Proxemic group behaviors using reciprocal multi-agent navigation, 2016 IEEE International Conference on Robotics and Automation (ICRA), 292--297.

7. S. Bandini, L. Crociani, A. Gorrini, and G. Vizzari, An agent-based model of pedestrian dynamics considering groups: A real world case study, IEEE 17th International Conference on Intelligent Transportation Systems (ITSC), 2014, 572--577.

8. N. Bode, S. Holl, W. Mehner, and A. Seyfried, Disentangling the impact of social groups on response times and movement dynamics in evacuations, PLoS one http://dx.doi.org/10.1371/journal.pone.0121227.

9. G. Köster, F. Treml, M. Seitz, and W. Klein, Validation of crowd models including social groups. In Ulrich Weidmann, Uwe Kirsch, and Michael Schreckenberg, editors, Pedestrian and Evacuation Dynamics 2012, pages 1051-1063. Springer International Publishing, 2014

10. X. Wei, X. Lv, W. Song, X. Li, Survey study and experimental investigation on the local behavior of pedestrian groups, Complexity, Volume 20, Issue 6, July/August 2015, Pages 87-97.

11. I. Karamouzas, and M. Overmars, Simulating the local behaviour of small pedestrian groups, Proceedings of the 17th ACM Symposium on Virtual Reality Software and Technology, 183-190, (2010)

12. Y. Zhang, J. Pettré, X. Qin, S. Donikian and Q. Peng, A Local Behavior Model for Small Pedestrian Groups, Computer-Aided Design and Computer Graphics (CAD/Graphics), 2011 12th International Conference on, 275-281 (2011)

13. A. Gorrini, L. Crociani, C. Feliciani, P. Zhao, K. Nishinari, S. Baldini, Social groups and pedestrian crowds: experiment on dyads in a counter flow scenario, Proceedings of the 2016 Pedestrian and Evacuation Dynamics Conference

14. M. Seitz, A. Templeton, J. Drury, G. Köster, and A. Philippides, Parsimony versus reductionism: how can crowd psychology be introduced into computer simulation?, Review of General Psychology, 21(1), 95, 2017

15. Z. Yücel, F. Zanlungo and M. Shiomi, Modeling the impact of interaction on pedestrian group motion Advanced Robotics, 1-11 (2018).

16. M. Costa, Interpersonal distances in group walking, Journal of Nonverbal Behavior, 34, 1, 15-26 (2010)

17. F. Zanlungo, Z. Yücel Z, D. Brščić, T. Kanda T and N. Hagita, Intrinsic group behaviour: Dependence of pedestrian dyad dynamics on principal social and personal features, PLoS One, 2017, 0187253

18. D. Bugental, Acquisition of the algorithms of social life: A domain-based approach, Psychological bulletin, 126, 2,187, 2000 